International Journal of Modern Physics B

Vol. 33, No. 22 (2019) 1950249 (14 pages)

(C) The Author(s)

DOI: $10.1142 / \mathrm{S} 0217979219502497$

\title{
Link prediction based on combined influence and effective path
}

\author{
Yang Tian*, Han $\mathrm{Li}^{*, \ddagger}$, Xuzhen Zhu ${ }^{\dagger}$ and Hui Tian ${ }^{\dagger}$ \\ * School of Applied Science, \\ Beijing Information Science and Technology University, \\ Beijing 100192, P. R. China \\ $\dagger$ State Key Laboratory of Networking and Switching Technology, \\ Beijing University of Posts and Telecommunications, \\ Beijing 100876, P. R. China \\ $\ddagger$ lh.li@bistu.edu.cn
}

Received 15 November 2018

Revised 24 May 2019

Accepted 15 July 2019

Published 29 July 2019

\begin{abstract}
Link prediction based on topological similarity in complex networks obtains more and more attention both in academia and industry. Most researchers believe that two unconnected endpoints can possibly make a link when they have large influence, respectively. Through profound investigations, we find that at least one endpoint possessing large influence can easily attract other endpoints. The combined influence of two unconnected endpoints affects their mutual attractions. We consider that the greater the combined influence of endpoints is, the more the possibility of them producing a link. Therefore, we explore the contribution of combined influence for similarity-based link prediction. Furthermore, we find that the transmission capability of path determines the communication possibility between endpoints. Meanwhile, compared to the local and global path, the quasi-local path balances high accuracy and low complexity more effectually in link prediction. Therefore, we focus on the transmission capabilities of quasi-local paths between two unconnected endpoints, which is called effective paths. In this paper, we propose a link prediction index based on combined influence and effective path (CIEP). A large number of experiments on 12 real benchmark datasets show that in most cases CIEP is capable of improving the prediction performance.
\end{abstract}

Keywords: Complex networks; link prediction; combined influence; effective path.

PACS numbers: 89.65.-5, 89.75.-k

This is an Open Access article published by World Scientific Publishing Company. It is distributed under the terms of the Creative Commons Attribution 4.0 (CC BY) License which permits use, distribution and reproduction in any medium, provided the original work is properly cited.

${ }^{\ddagger}$ Corresponding author. 


\section{Introduction}

In the past few years, many researchers explored the potential or missing links in complex networks, $, 2,2$ which can be described with nodes and relations between two unconnected endpoints. Especially, link prediction based on the topological similarity ${ }^{3-6}$ becomes the focus of the research fields. In the aspect of practical applications, link prediction can be widely used to do research on the relationships between proteins in the biological ecosystems,,$\frac{7,8}{2}$ the potential relationships in the social networks,,$\frac{9}{-12}$ the user-commodity relationships in the e-commerce networks, $\frac{13}{,}$ the related information in the collaborative information filtering systems, $\stackrel{14}{-16}$ the infrastructure planning in the transportation networks, the route planning in the aviation networks, ${ }^{17,18}$ the optimal resource diffusion for suppressing disease spreading in the multiplex networks ${ }^{19}$ and the applications in the wireless sensor networks, ${ }^{20}$ etc.

Driven by a lot of researchers, the topological similarity-based link prediction achieves a great success. The topological similarity assumes that the sufficient resources and the strong transmission capabilities of paths will facilitate the potential connection between two unconnected endpoints. According to the length of paths, the topological similarity indices can be divided into three categories: local, global and quasi-local indices. The local similarity indices based on the topological structure will promote the possibility of generating a connection between two unconnected endpoints. For example, Common Neighbors index $(\mathrm{CN})^{21,22}$ considers the number of CNs. Adamic-Adar index (AA) ${ }^{23,24}$ and Resource-Allocation index $(\mathrm{RA})^{25,26}$ depress the common neighbor nodes with large degree. Different from the above indices, Sørensen index ${ }^{27}$ and Leicht-Holme-Newman index (LHN-I) ${ }^{28}$ penalize the endpoints with large degree. Katz index, ${ }^{29}$ a global similarity index, involves all paths between two unconnected endpoints and prefers the short paths. To optimize the low prediction accuracy of the local similarity indices and the high computational complexity of the global similarity indices, researchers propose many quasi-local similarity indices. ${ }^{30,31}$ For example, Local Path index (LP) ${ }^{25,30}$ considers the number of all two-step and three-step paths with the number of all two-step paths preferred. Effective Path index (EP) ${ }^{3}$ uses the effective influence of endpoints and the strong connectivity in similarity calculation. Nonequilibrium Cooperation Effect index (NCE) ${ }^{32}$ describes the large-degree heterogeneity as the nonequilibrium which facilitates a stable cooperation between endpoints. In recent years, for further improving the performance of link prediction, some indices using the path information continue to be proposed. For example, Yao et al. ${ }^{33}$ propose a Resources from Short Paths index (RSP). It considers the interactions among the paths with different length and adopts the resource-traffic flow mechanism to measure the interactions among the paths between node pairs. $\mathrm{Xu}$ et al. ${ }^{34}$ propose a Weighted Path Entropy index (WPE) to improve the prediction accuracy in the weighted networks. It uses information entropy to measure the contribution of a path in link prediction and further quantifies the contribution of a path with both 
path entropy and path weight. Shakibian et al. $\frac{35}{3}$ propose a multi-layered approach in which a heterogeneous system is modeled as a multi-layered complex network. Then, through exploring the network layers with different semantics, a set of metapaths is generated. Extracting a number of topological features for each meta-path, a number of link predictors is learned which are aggregated to build the final link predictor.

Until now, existing link prediction indices consider that two unconnected endpoints with large influence can possibly make a link. However, through further research, we find that the total influence resources of two unconnected endpoints are mutually transferred on a path. The attractions of two unconnected endpoints depends on their combined influence instead of single one. We believe that the greater the combined influence is, the more the possibly of two unconnected endpoints producing a link. Therefore, we explore the contribution of the combined influence for similarity between two unconnected endpoints. To more intuitively explain the role of the combined influence, we give an illustration in Fig. 1. In Fig. 1, degree is 5, 5 in endpoints a1 and a5, and 2, 10 in endpoints b1 and b5, respectively. Compared to endpoints b1 and b5, endpoints a1 and a5 have larger influence, respectively. Nevertheless, only the influence of single endpoint cannot affect the attractions of two unconnected endpoints. In complex networks, the influence resources of two unconnected endpoints are transferred between each other through all their paths. In other words, the total influence resources are passed on the same path. Through careful investigation, we believe that the combined influence determines the mutual attractions of two unconnected endpoints. Therefore, the sum of degree can more accurately evaluate the similarity of two unconnected endpoints. For example, in the condition that a 1 and a 5 have degree $=5$ and 5 and b1 and b5 have degree $=$ 2 and 10, the sum of degree is 10 of a1 and a5, and 12 of b1 and b5. Compared to a1 and a5, b1 and b5 jointly have greater combined influence. In a word, b1 and b5 have the most possibility to make a link in the future.

To illustrate the important role of the combined influence, we give four examples. Firstly, American president has a huge influence as a public figure in the world. People who contact him include not only human with the same influence, but

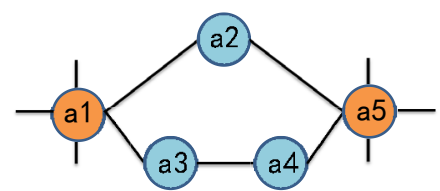

(a)

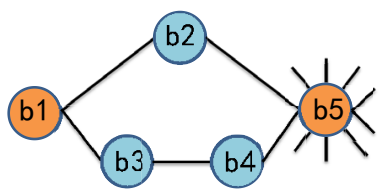

(b)

Fig. 1. (Color online) Illustration of the combined influence in complex networks. Subgraph (a) shows that the endpoints a1 and a5 have degree $=5$ and 5 , respectively. Subgraph (b) shows that the endpoints b1 and b5 have degree $=2$ and 10, respectively. Subgraphs (a) and (b) show that the influence resources of two unconnected endpoints are transferred to each other through their every path, respectively. 
also common person, such as his ordinary relatives or friends. Secondly, a known professor with significant achievements has a great influence in academia. People who contact him include not only professors with the same influence, but also common person, such as his/her ordinary students. Thirdly, a known entrepreneur with great wealth has a great influence in commercial circles. When he/she invests more money in a football club for his/her own preference or business interests, people who contact him include not only businessmen with the same status, but also these ordinary football players in his/her club. Lastly, as a star in one field, he/she obtains more worship of ordinary fans. His/her twitter can attract more and more attentions of ordinary fans.

In this paper, we leverage the combined influence of endpoints and the transmission capability of quasi-local path to construct our model, namely CIEP. Through deep investigation and extensive experiments, we find that in many cases CIEP is capable of improving the prediction accuracy.

The rest of this paper is organized as follows. In Sec. 2, we structure a link prediction model. In Secs. 3 and 4, we show the 12 benchmark experimental datasets and methods, including a metric and eight mainstream baselines. In Sec 5, we present the results and discussions. Finally, we provide a conclusion in Sec. 6 .

\section{Model Based on Combined Influence and Effective Path}

In this section, we define the problem definition in Sec. 2.1, propose the combined influence model, the transmission capability model and CIEP model in Sec. 2.2.

\subsection{Problem definition}

Complex network analysis should model the networks and define several metrics for analyzing experimental results. An undirected simple network can be modeled as a graph $G=(V, E)$, where $E=\bigcup_{i} E_{i}$ and $V=\bigcup_{i} V_{i}$ are the set of nodes and the set of links, respectively. Multiple links and self-connections are eliminated. We represent an interaction between nodes $x$ and $y$ as $e=(x, y) \subset E$. Each nonexistent link is modeled as $(x, y) \subset U-E$, where $x, y \subset V$ and $U$ represent the universal set. Each link prediction algorithm assigns a score value $s_{x y}$ to each nonexistent link to qualify its likelihood of existence. The similarity between nodes $x$ and $y$ is defined by a score and a higher score means a higher probability of the connection between $x$ and $y$.

\subsection{CIEP model}

In complex networks, two unconnected endpoints possessing small influence hardly produce a link. However, at least one endpoint with large influence can easily attract other endpoints. Therefore, the mutual attractions between two unconnected endpoints depend on their combined influence. Furthermore, the influence resources of endpoints are passed between each other by paths connecting two unconnected 
endpoints. The transmission capability of path determines the communication possibility between endpoints. Meanwhile, compared to the local and global path, the quasi-local path balances high accuracy and low complexity more effectually in link prediction. Therefore, we propose a link prediction method considering the combined influence and the transmission capability.

Definition 1. On an undirected unweighted network $G=(V, E)$, a node set $P_{l}=\left\{n_{0}=x, n_{1}, \ldots, n_{l-1}, n_{l}=y\right\}$ constitutes the length of paths $l$ between two unconnected endpoints $x$ and $y . k_{x}$ and $k_{y}$ represent the degree of $x$ and $y$, respectively. The similarity of $x$ and $y$ depends on their combined influence because their total influence resources are mutually passed on a path. Therefore, the combined influence $\mathrm{CI}(x, y)$ equals the sum of influence resources of $x$ and $y$ as

$$
\mathrm{CI}(x, y)=\frac{k_{x}+k_{y}}{2|E|}
$$

where $\frac{k_{x}}{2|E|}$ and $\frac{k_{y}}{2|E|}$ represent the influence recourses of $x$ and $y$, respectively, and $|E|$ indicates the number of connected links of the network.

Definition 2. On an undirected unweighted network $G=(V, E)$, the transmission capabilities $\left.\mathrm{TC}(x, y)\right|_{l} ^{2}$ of all two-step paths between $x$ and $y$ equal the sum of the reciprocal of the node degree from $x$ to $y$ or from $y$ to $x$ as

$$
\left.\mathrm{TC}(x, y)\right|_{l} ^{2}=\sum_{i \in p_{2}(x, y)} \frac{1}{k_{i}},
$$

and the transmission capabilities $\left.\mathrm{TC}(x, y)\right|_{l} ^{3}$ of all three-step paths as

$$
\left.\mathrm{TC}(x, y)\right|_{l} ^{3}=\sum_{i, j \in p_{3}(x, y)} \frac{1}{k_{i} k_{j}} .
$$

Synthetically, the total transmission capabilities $\mathrm{TC}(x, y)$ of quasi-local paths between $x$ and $y$ can be denoted as

$$
\mathrm{TC}(x, y)=\sum_{i \in p_{2}(x, y)} \frac{1}{k_{i}}+\varepsilon \sum_{i, j \in p_{3}(x, y)} \frac{1}{k_{i} k_{j}},
$$

where $\frac{1}{k_{i}}$ indicates the similarity transferability of intermediate node $n_{i}$, with $k_{i}$ denoting the intermediate node degree, and $\varepsilon$ in $(0,1)$ serves as a tunable parameter suppressing the contributions of all three-step paths for the strong connectivity.

Definition 3. On an undirected unweighted network $G=(V, E)$, integrating the combined influence model $\mathrm{CI}(x, y)$ and the transmission capability model $\mathrm{TC}(x, y)$, the similarity model $s_{x y}^{\mathrm{CIEP}}(\varepsilon)$ can be defined as

$$
\begin{aligned}
s_{x y}^{\mathrm{CIEP}}(\varepsilon) & =\mathrm{CI}(x, y) \times \operatorname{TC}(x, y) \\
& =\frac{k_{x}+k_{y}}{2|E|}\left(\sum_{i \in p_{2}(x, y)} \frac{1}{k_{i}}+\varepsilon \sum_{i, j \in p_{3}(x, y)} \frac{1}{k_{i} k_{j}}\right) .
\end{aligned}
$$




\section{Experimental Data}

To verify the performance of model, we apply abundant experiments on 12 benchmark networks. A detailed description of the 12 benchmark networks exhibits as follows: (1) US Air97 (USAir) ${ }^{36}$ represents the network of the US air transportation system; (2) Yeast PPI (Yeast) ${ }^{37}$ shows the network composed of protein interactions of yeast; (3) Food Web of Florida ecosystem (Food) ${ }^{38}$ contains the relationship of carbon exchanges in the cypress wetlands of South Florida during the wet season; (4) Power Grid (Power) ${ }^{39}$ represents the network of the western US power grid, describing high voltage transmission among generators, transformers and substations; (5) Net Science (NS) ${ }^{40}$ represents the network of co-authorships between scientists publishing on the topic of networks; (6) Jazz ${ }^{41}$ denotes the network of Jazz musicians; (7) Email network (Email) ${ }^{42}$ is the email communication network of University Rovira i Virgili (URV) in Tarragona, Spain; (8) Slavko ${ }^{43}$ indicates the Facebook friendship network of Slavko Zitnik; (9) UC Irvine messages social network (Ucsocial $)^{44}$ is the communication network constructed by the messages between users of an online community of students from the University of California, Irvine; (10) Infectious (Infec) ${ }^{45}$ indicates the face-to-face contact network of people during the exhibition "Infectious: Stay Away" in 2009 at the Science Gallery in Dublin; (11) EuroSiS web (EuroSiS) ${ }^{46}$ maps interactions between actors in Science of Society on the network of 12 European countries; (12) C.elegans (CE) ${ }^{39}$ represents the neural network of the nematode worm CE. The basic topological characteristics of the above networks are shown in Table 1.

To test the performances of the models, we divide the set of links $E$ randomly into two parts: a training set, $E^{T}$, containing $90 \%$ of the known links, and a testing set, $E^{P}$, containing the remaining $10 \%$. On each dataset, we run 50 independent random divisions and obtain the averaged prediction results.

Table 1. The basic topological features of the 12 benchmark networks. There are the properties: $|V|$ denoting the number of nodes, $|E|$ denoting the number of links, $\langle k\rangle$ denoting the average degree, $\langle d\rangle$ representing the average distance, $C$ representing the clustering coefficient, $r$ indicating the assortativity coefficient and $H$ indicating the degree heterogeneity and defined as $H=\frac{\left\langle k^{2}\right\rangle}{\langle k\rangle^{2}}$.

\begin{tabular}{lrrrrrrr}
\hline \multicolumn{1}{c}{ Nets } & $|V|$ & $|E|$ & $\langle k\rangle$ & $\langle d\rangle$ & $C$ & \multicolumn{1}{c}{$r$} & $H$ \\
\hline USAir & 332 & 2128 & 12.81 & 2.74 & 0.749 & -0.208 & 3.36 \\
Yeast & 2370 & 10904 & 9.2 & 5.16 & 0.378 & 0.469 & 3.35 \\
Food & 128 & 2075 & 32.42 & 1.78 & 0.334 & -0.112 & 1.24 \\
Power & 4941 & 6594 & 2.669 & 15.87 & 0.107 & 0.003 & 1.45 \\
NS & 1461 & 2742 & 3.75 & 5.82 & 0.878 & 0.461 & 1.85 \\
Jazz & 198 & 2742 & 27.7 & 2.24 & 0.633 & 0.02 & 1.4 \\
Email & 1133 & 5451 & 9.62 & 3.61 & 0.254 & 0.078 & 1.94 \\
Slavko & 334 & 2218 & 13.28 & 3.05 & 0.488 & 0.247 & 1.62 \\
Ucsocial & 1893 & 13825 & 14.62 & 3.06 & 0.138 & -0.188 & 3.81 \\
Infec & 410 & 2765 & 13.49 & 3.63 & 0.467 & 0.226 & 1.39 \\
EuroSiS & 1272 & 6454 & 10.15 & 3.86 & 0.382 & -0.012 & 2.46 \\
CE & 453 & 2025 & 8.94 & 2.66 & 0.655 & -0.225 & 4.49 \\
\hline
\end{tabular}




\section{Experimental Methods}

\subsection{Metric}

AUC,, 47 a metric of accuracy, can be interpreted as the probability that a potential link (a link in $E^{P}$ ) ranks a higher score than a nonexistent link (a link in $U \backslash E$, where $U$ denotes the universal link set). In the specific implementation, among $n$ independent comparisons, if the potential link ranks higher in $n^{\prime}$ times and the same as the nonexistent link in $n^{\prime \prime}$ times, the total score accumulates $n^{\prime}$ and $0.5 n^{\prime \prime}$. After that, AUC expresses the averaged score over $n$-time comparisons as

$$
\mathrm{AUC}=\frac{n^{\prime}+0.5 n^{\prime \prime}}{n} .
$$

AUC evaluates the performance of a model globally. If all scores originate from an independent and identical distribution, the value should equal to 0.5 . Therefore, the extent to which the accuracy exceeds 0.5 suggests how much better a model performs than pure chance.

\subsection{Baselines}

For comparisons, we introduce eight classical models as follows:

(1) CN (Common Neighbors index) ${ }^{21,22}$ calculates the similarity by counting the number of common neighbors with the definition as

$$
s_{x y}^{\mathrm{CN}}=|\Gamma(x) \cap \Gamma(y)|,
$$

where $\Gamma(x)$ and $\Gamma(y)$ represent the set of neighboring nodes belonging to the endpoints $x$ and $y$, respectively, and $\Gamma(x) \cap \Gamma(y)$ denotes the set of common neighbors of the endpoints $x$ and $y$.

(2) AA (Adamic-Adar index) ${ }^{23,24}$ punishes the common neighbors with big degree by considering the logarithm of reciprocal of common neighbors' degrees. So, based on $\mathrm{CN}$, it uses the reciprocal of logarithm to suppress common neighbors with big degree, defined as

$$
s_{x y}^{\mathrm{AA}}=\sum_{z \in \Gamma(x) \cap \Gamma(y)} \frac{1}{\log \left(k_{z}\right)},
$$

where $k_{z}$ denotes the node degree of $z$.

(3) RA (Resource-Allocation index) $)^{25,26}$ uses the reciprocal of degree to suppress $\mathrm{CN}$ with big degree, defined as

$$
s_{x y}^{\mathrm{RA}}=\sum_{z \in \Gamma(x) \cap \Gamma(y)} \frac{1}{k_{z}} .
$$

(4) PA (Preference Attachment index) ${ }^{21,48}$ leverages the preferential attachment phenomena rule discovered in a variety of social networks. In this method, the 
similarity score is equivalent to the product of the degrees of the involved nodes, defined as

$$
s_{x y}^{\mathrm{PA}}=k_{x} k_{y} .
$$

(5) Salton ${ }^{49}$ counts the number of common neighbors by considering the geometric averages of node degree and normalizes the CN using $\sqrt{k_{x} k_{y}}$, defined as

$$
s_{x y}^{\text {Salton }}=\frac{|\Gamma(x) \cap \Gamma(y)|}{\sqrt{k_{x} k_{y}}} .
$$

(6) Sørensen ${ }^{27}$ counts the number of common neighbors by considering the arithmetic averages of node degree. A model based on the similarity of species content is established in plant sociology, defined as

$$
s_{x y}^{\mathrm{S} \varnothing \mathrm{rensen}}=\frac{2 \times|\Gamma(x) \cap \Gamma(y)|}{k_{x}+k_{y}} .
$$

(7) LHN-I (Leicht-Holme-Newman index) ${ }^{28}$ counts the number of common neighbors by considering the square of the geometric averages of node degree. The algorithm provides high similarities to pairs of nodes that have many common neighbors, defined as

$$
s_{x y}^{\mathrm{LHN}-\mathrm{I}}=\frac{|\Gamma(x) \cap \Gamma(y)|}{k_{x} k_{y}} .
$$

The denominator $k_{x} k_{y}$ is proportional to the expected value of the number of common neighbors for the endpoints $x$ and $y$.

(8) LP (Local Path index) ${ }^{25,30}$ considers the quasi-local paths of lengths 2 and 3 with the paths of length 2 preferred, defined as

$$
S^{\mathrm{LP}}=A^{2}+\varepsilon A^{3}
$$

where $A$ and $\varepsilon$ denote the adjacency matrix and the free punishment parameter, respectively.

\section{Results and Discussion}

In order to verify the performances of CIEP, we conduct considerable experiments on the 12 benchmark datasets. Through comparisons with several mainstream baselines in terms of accuracy metric AUC, we obtain the accuracy performance results and discuss the findings in the following. For clarifying the dependence of accuracy on value of tunable parameter $\varepsilon$, we plot the relation curves in Fig. 2.

Figure 2 depicts the average AUC curve with different $\varepsilon$ over 50 independent random simulations in the 12 datasets with disparate topological characteristics. All AUC curves reach the optimal peaks with each certain $\varepsilon$ value in $(0,1)$, i.e., 0.4 in USAir, 0.5 in Yeast, 0.3 in Food, 0.1 in Power, 0.7 in NS, 0.2 in Jazz, 0.2 in Email, 0.2 in Slavko, 0.1 in Ucsocial, 0.9 in Infec, 0.1 in EuroSiS, and 0.2 in CE. Illustrated in Fig. 2, the obtained optimal AUC results in all datasets show the 

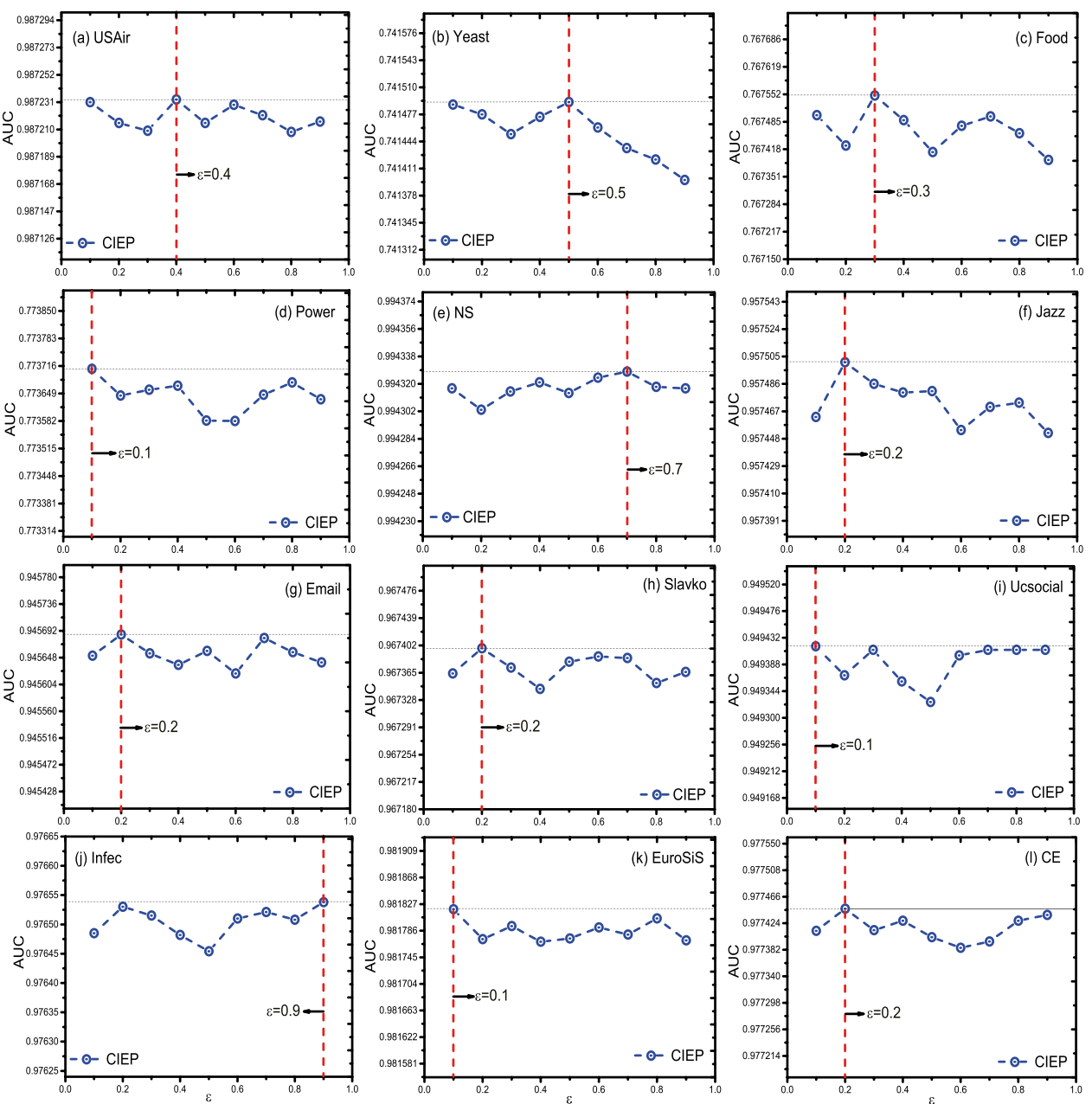

Fig. 2. (Color online) Results of AUC fluctuating with the parameter $\varepsilon$, even though all datasets have different topological properties. AUC values are averages over 50 independent random simulations.

role of combined influence of endpoints and transmission capabilities of quasi-local paths.

To make intuitive comparisons, we plot Fig. 3 to show the dependence of AUC on the tunable parameter $\varepsilon$ for CIEP and LP. In Fig. 3, there exists a certain parameter $\varepsilon$ to obtain their optimal AUC results for CIEP and LP. CIEP achieves higher accuracy than LP in the 11 datasets, including USAir, Yeast, Power, NS, Jazz, Email, Slavko, Ucsocial, Infec, EuroSiS and CE, meaning that our proposed similarity index can achieve the higher prediction accuracy. Therefore, we prove the positive contributions of the combined influence and the transmission capability for link prediction. 

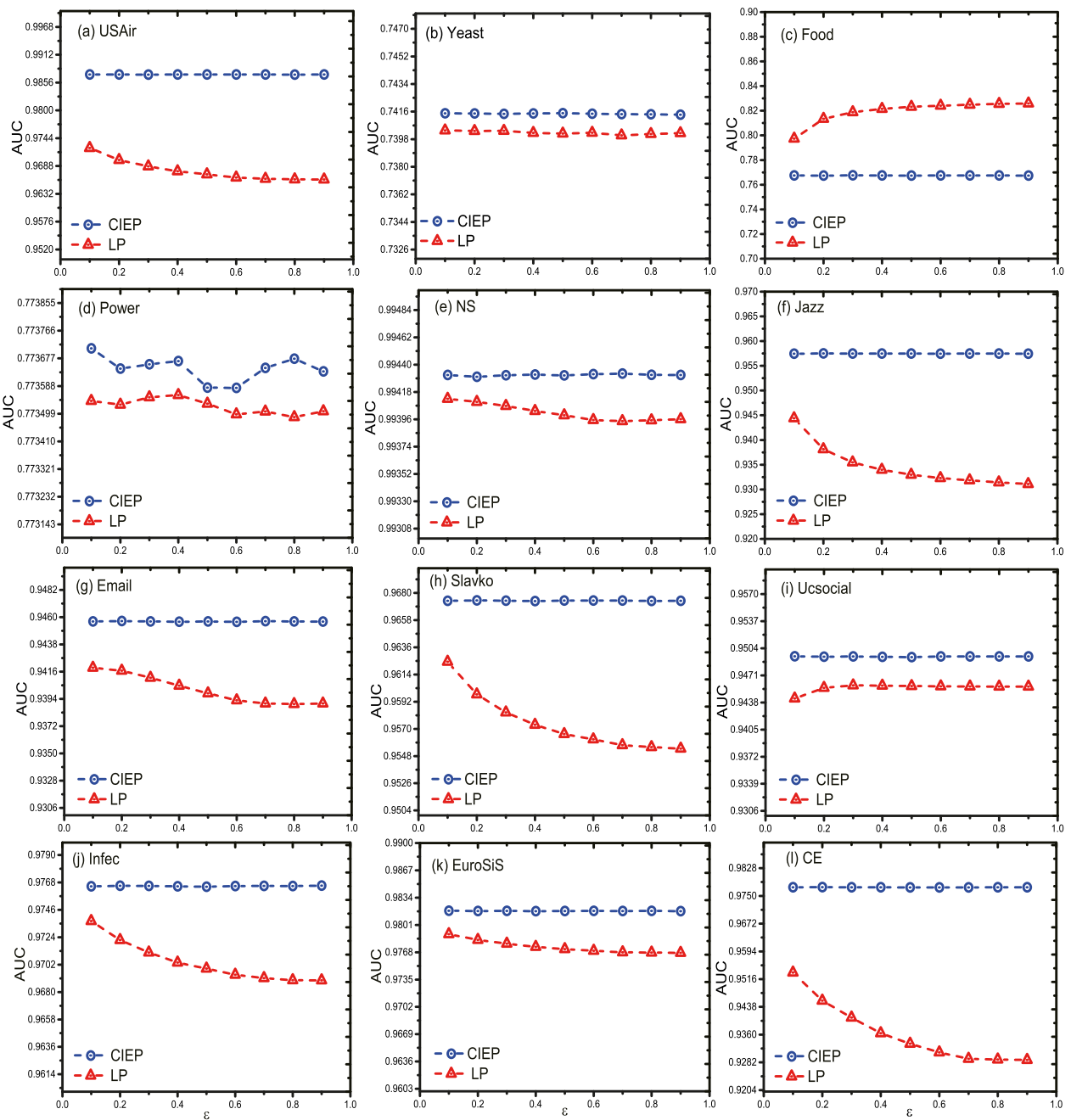

Fig. 3. (Color online) The above diagram shows a dependence illustration of the metric AUC on the parameter $\varepsilon$. The relation curves between AUC and $\varepsilon$ in terms of CIEP and LP are provided on the 12 datasets. On each dataset, CIEP and LP identically obtain the optimal AUC performances at a certain $\varepsilon$. In this paper, the division of the training and testing set is independent random in 50 times. And the results are the averages of the 50 simulations.

We compare CIEP with the mainstream link prediction indices: CN, AA, RA, PA, Salton, Sørensen, LHN-I and LP. For clearly exhibiting the results, we give the averaged AUC values over 50 independent simulation realizations for all indices in Table 2. The bold fonts emphasize the optimal performance in each dataset, and the numbers in parenthesis indicate the obtained certain parameter values for CIEP and LP. Table 2 shows that CIEP obtains most of the optimal AUC performances.

In Table 2, CIEP outperforms eight mainstream baselines (referring to the values in bold font), especially outstanding in Infec and EuroSiS. To investigate the 


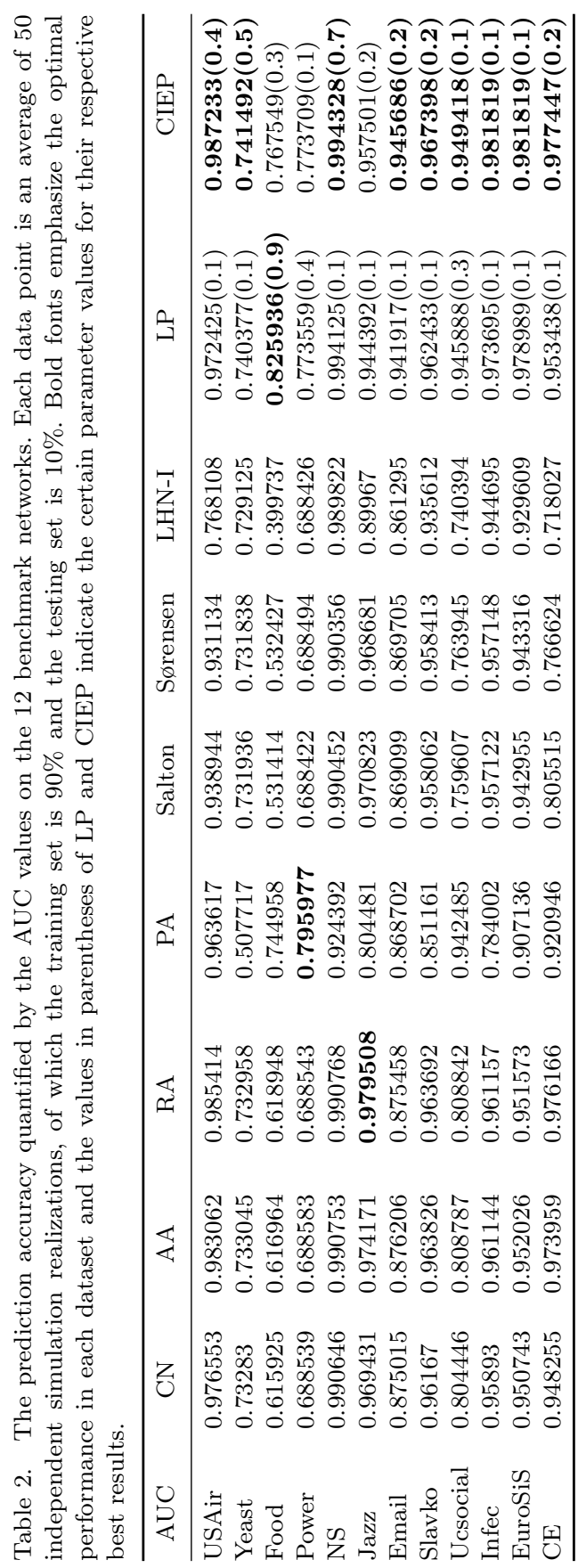


underlying reason, the combined influence and the transmission capability jointly reveal the difference. CN considers the number of common neighbors, which is equivalent to the number of two-step paths between two unconnected endpoints, but ignores the influence of endpoints, leading to the worst performances, such as in Email and Ucsocial. Different from CN, considering the influence of endpoint based on common neighbors, Salton, Sørensen and LHN-I are generated in different ways from different perspectives. However, they only focus on the influence of single endpoint and ignore the combined influence of two unconnected endpoints, resulting in poor accuracy. With a little improvement in contrast to $\mathrm{CN}, \mathrm{AA}$ and RA consider the connectivity of all two-step paths, but also neglect the combined influence of endpoints. Furthermore, LP considers the influence of single endpoint and contributions of the quasi-local paths, but ignores the combined influence of two unlinked endpoints. Above all, CIEP conquers the weaknesses of baselines and considers the combined influence of endpoints based on the quasi-local paths, bringing in an excellent self-adaptive link prediction performances on most of the networks with complicated topological characteristics.

Besides, the low computation complexity is a significant concern in the construction of link prediction model. Generally, the time complexity of product of two $N \times N$ matrices is $O\left(N^{3}\right)$. According to the definitions, CN, AA, RA, PA, Salton, Sørensen and LHN-I all have the time complexity of $O\left(N^{3}\right)$ and LP has the time complexity of $M \times O\left(N^{3}\right)$ with coefficient $M \ll N^{3}$. In contrast, although with same time complexity of $M \times O\left(N^{3}\right)$ with LP, more than CN, AA, RA, PA, Salton, Sørensen and LHN-I, CIEP shows better performances. Above all, CIEP achieves the best performances without increasing the time complexity.

\section{Conclusions}

Researchers contribute to link prediction and obtain fruitful achievements, especially the findings based on the topological similarity of the complex networks. With respect to topological similarity, the traditional researches ignore the combined influence of endpoints and the transmission capabilities of quasi-local paths which can enhance the link prediction accuracy. Therefore, through deep researches, we propose a CIEP index. Comparisons between CIEP and CN, AA, RA, PA, Salton, Sørensen, LHN-I and LP on the 12 datasets confirm that CIEP acquires a considerable improvement in prediction accuracy. Besides, CIEP brings in no increase in complexity.

Compared to traditional researches, CIEP improves the link prediction performance and has strong adaptability on different datasets. It is a heuristic method and provides a reference for future research. We propose CIEP index by considering the combined influence and the transmission capability for improving link prediction performance. The experimental results on complex networks show that CIEP achieves high prediction accuracy and does not increase complexity. However, we only consider the degree as the influence recourses of endpoints. Actually, the 
degree cannot completely represent the influence resources. Coreness and H-index also can play an important roles for the influence of endpoints. Furthermore, the proposed method in this paper focuses on the length and the transmission capability of path. However, the intermediate nodes of path also play an important role for the similarity between endpoints. Our limitation is the evaluation approaches of the endpoints' influence and the path's heterogeneity. We will further study the contributions of the endpoints' influence and the path's heterogeneity for link prediction in future research. In addition, our research may facilitate the development of future link prediction in practical applications, such as in recommendation systems, dynamic systems, social communication systems, collaborative information filtering systems, ecological systems, sensor systems and so on.

\section{Acknowledgments}

This work is partially supported by the Scientific Research Program of Beijing Municipal Education Commission (KM201511232019) and the National Natural Science Foundation of China (Grant Nos. 61471060 and 61602048).

\section{References}

1. L. Lü et al., Phys. A. Stat. Mech. Appl. 390, 1150 (2011).

2. L. Lü et al., Complex Syst. Complex. Sci. 7, 173 (2010).

3. X. Zhu et al., Phys. A. Stat. Mech. Appl. 413, 515 (2014).

4. W. Cui et al., Phys. A. Stat. Mech. Appl. 457, 202 (2016).

5. E. Bastami et al., Swarm Evol. Comput. 44, 176 (2019).

6. Y. Weiwei et al., Inf. Fusion 46, 1 (2019).

7. M. Kurakar et al., Int. J. Comput. Trends Technol. 9, 164 (2014).

8. K. V. Singh et al., Appl. Netw. Sci. 2, 2 (2017).

9. B. Chang et al., Int. J. Autom. Comput. 15, 3 (2018).

10. Y. Xiao et al., Sci. China Inf. Sci. 61, 48 (2018).

11. M. Mochon et al., Int. J. Int. Multimedia Artif. Intell. 3, 34 (2016).

12. W. Wang et al., Phys. A. Stat. Mech. Appl. 499, 121 (2018).

13. Y. Wu et al., Phys. A. Stat. Mech. Appl. 502, 288 (2018).

14. F. Zhao et al., A filtering approach based on MMAE for a SINS/CNS integrated navigation system, in American Control Conf. (IEEE, Seattle, 2017).

15. Y. Liu et al., Big Data Min. Anal. 1, 211 (2018).

16. Z. Yan et al., Glob. Energy Interconnect. 5, 559 (2018).

17. X. Wen et al., Phys. A. Stat. Mech. Appl. 503, 546 (2018).

18. H. Li et al., Int. J. Disaster Risk Sci. 3, 45 (2016).

19. X. Chen et al., J. Stat. Mech. Theory Exp. 2018, 053501 (2018).

20. X. Luo et al., J. Syst. Eng. Elect. 29, 51 (2018).

21. M. E. J. Newman, Phys. Rev. E 64, 025102 (2001).

22. Y. Liu et al., Phys. A. Stat. Mech. Appl. 454, 24 (2016).

23. L. A. Adamic et al., Soc. Netw. 25, 211 (2003).

24. H. Yao et al., J. Syst. Sci. Inf. 5, 446 (2017).

25. T. Zhou et al., Eur. Phys. J. B 71, 623 (2009).

26. A. N. Letchford et al., Comput. Optim. Appl. 68, 193 (2017).

27. T. Sørensen, Biol. Skrif. 5, 1 (1948). 
28. E. A. Leicht et al., Phys. Rev. E 73, 026120 (2006).

29. L. Katz, Psychometrika 18, 39 (1953).

30. L. Lü et al., Phys. Rev. E 80, 046122 (2009).

31. X. Wang et al., Phys. A. Stat. Mech. Appl. 419, 260 (2015).

32. L. Li et al., Int. J. Mod. Phys. B 32, 1850128 (2018).

33. Y. Yao et al., Phys. A. Stat. Mech. Appl. 52, 52 (2018).

34. Z. H. Xu et al., Chinese Phys. B 1, 588 (2017).

35. H. Shakibian et al., Phys. A. Stat. Mech. Appl. 501, 248 (2018).

36. V. Batagelj et al., Connections 21, 47 (1998).

37. D. Bu et al., Nucleic Acids Res. 31, 2443 (2003).

38. C. Melian et al., Ecology 85, 352 (2004).

39. D. J. Watts et al., Nature 393, 440 (1998).

40. M. E. J. Newman, Phys. Rev. E 74, 036104 (2006).

41. P. M. Gleiser et al., Adv. Complex Syst. 6, 565 (2003).

42. R. Guimera et al., Phys. Rev. E 68, 065103(R) (2003).

43. T. Opsahl et al., Soc. Netw. 31, 155 (2009).

44. N. Blagus et al., Phys. A. Stat. Mech. Appl. 391, 2794 (2012).

45. L. Isella et al., J. Theor. Biol. 271, 166 (2010).

46. D. Van Welden, Mapping system theory problems to the field of knowledge discovery in databases, in FUBUTEC'2004:1st Future Bus. Technol. Conf., Vol. 55 (Fontainebleau, France, 2004).

47. M. Kitsak et al., Nat. Phys. 6, 888 (2010).

48. Y. Xie et al., Phys. A. Stat. Mech. Appl. 387, 1683 (2008).

49. M. Davenport, Education for Information. 2, 221 (2000). 